\title{
Acoustic Analysis of Voice Change in Normal Speakers Following Transcutaneous Electrical Stimulation to the Laryngeal Area
}

\author{
Mary Gorham-Rowan ${ }^{*}, 1$, Linda Fowler ${ }^{2}$ and Edie Hapner ${ }^{3}$ \\ ${ }^{I}$ Department of Communication Sciences and Disorders, Valdosta State University, Valdosta, GA, USA \\ ${ }^{2}$ Communication Disorders Program, Georgia State University, Atlanta, GA, USA \\ ${ }^{3}$ Emory Voice Center, Emory University, Atlanta, GA, USA
}

\begin{abstract}
Transcutaneous electrical stimulation (TES) is being used more frequently by speech-language pathologists to treat individuals with dysphagia since the advent of VitalStim ${ }^{\circledR}$. Anecdotal reports suggest changes in voice quality associated with TES. This study was completed to examine acoustic measures of voice change in normal speakers following TES. Thirty speakers with normal voices, ages 19-59, received 1 hour of TES administered via two pairs of surface electrodes on the anterior neck. Voice recordings were obtained before and after TES, as well as subjective reports post-TES. The results of the study revealed nonsignificant changes in the acoustic voice measures following TES; these changes were highly variable. Participants reported no sensation, a feeling of vocal warm-up, vocal fatigue and/or delayed onset muscle soreness following TES. Some factors that may contribute to these findings include the amount of subcutaneous fat in the laryngeal area and attempts to resist hyolaryngeal lowering associated with TES.
\end{abstract}

Keywords: Voice, acoustic, transcutaneous electrical stimulation.

\section{INTRODUCTION}

The use of transcutaneous electrical stimulation (TES) has become more widespread among speech-language pathologists since the introduction of VitalStim ${ }^{\circledR}$, a portable transcutaneous neuromuscular device (The Chattanooga Group, Hixon, TN). TES has been approved by the FDA to treat individuals with pharyngeal dysphagia or swallowing dysfunction, and has been shown to improve swallowing function in individuals with pharyngeal dysphagia $[1,2]$. Conflicting evidence exists regarding its effectiveness, however, as some studies have documented no advantage of TES in comparison to traditional treatment procedures [3]. The contradictory evidence is likely due to a number of factors, including patient characteristics, specific swallowing deficits, co-treatment administration, etc.

Emerging anecdotal reports suggest that TES is also being used to treat individuals with disorders other than dysphagia, including voice and motor speech deficits. A few published reports have also documented improvement in vocal function. LaGorio, Carnaby-Mann, \& Crary [4] reported an improvement in maximum phonation time and pitch range in a patient who received TES in conjunction with traditional dysphagia therapy. They noted that the improvement in vocal function likely reflected the combined effect of TES and swallowing exercises e.g. an effortful swallow that the patient completed. While the use of an effortful swallow has been shown to improve laryngeal elevation and closure during swallowing in nondysphagic

*Address correspondence to this author at the Department of Communication Sciences and Disorders, Valdosta State University, 1500 North Patterson Street, Valdosta, GA 31698, USA; Tel: 229-219-1321;

E-mail:mmgorhamrowan@valdosta.edu individuals [5, 6], LaGorio et al. also suggested that application of TES to the anterior neck recruited intrinsic laryngeal muscle fibers, specifically the cricothyroid muscle, thus contributing to increased vocal fold tension and improved glottal closure. However, prior reports have not confirmed the activation of intrinsic laryngeal musculature with TES $[2,7]$. In a subsequent study, Humbert, Poletto, Saxon, Kearney, \& Ludlow [8] reported a slight change in the angle of the vocal folds with TES, but did not consider this change to be clinically meaningful. Given that prior reports have demonstrated changes in glottal opening associated with stimulation of strap musculature $[9,10]$, the extent to which TES may affect vocal function is not known.

To investigate the impact of TES upon voice, Fowler, Gorham-Rowan, \& Hapner [11] measured changes in fundamental frequency $\left(\mathrm{F}_{0}\right.$; the average number of vocal fold vibratory cycles per second) and vocal loudness in nondysphonic volunteers following a one-hour simulated treatment session of TES. While the results of the study revealed changes in both $\mathrm{F}_{0}$ and loudness, the direction and magnitude of change was variable across participants. Fowler et al. suggested that these voice changes likely reflected recruitment of extrinsic laryngeal muscle fibers, given that the low level of current amplitude used by the participants would not have been sufficient to stimulate cricothyroid activity [7, 8]. Furthermore, Fowler et al. suggested that the changes in $\mathrm{F}_{0}$ and/or loudness reflected a loading response by the laryngeal muscles, such as would occur following a period of prolonged talking.

The current study is an extension and replication of the previous work by Fowler et al. [11]. The purpose of this study was to examine changes in common acoustic measures in a larger group of patients following an hour of TES. In the previous study by Fowler et al., only $\mathrm{F}_{0}$ and relative sound level 
were examined. These parameters may be affected by extrinsic muscle contraction as well as intrinsic muscle activity $[10,12]$. Examining measures of perturbation and noise in the voice signal, which are presumed to reflect vocal fold activity, may provide a clearer picture of the effect of TES upon laryngeal function. Such measures would include jitter and shimmer, which are indicative of cycle-to-cycle variability in frequency and loudness, respectively [13]. A ratio of the amount of noise in vocal signal as compared to the acoustic energy, such as signal-to-noise ratio (SNR), may also prove useful. SNR is defined as the "decibel ratio of the total energy in the acoustic speech signal to the energy in the aperiodic, or noise component" [14]. Thus, SNR may provide additional information concerning vocal fold function associated with TES.
Although SNR has not been previously studied relative to the effects of prolonged voice use, or vocal loading, both jitter and shimmer have been shown to decrease in female teachers' voices at the end of a working day [15]. Since teaching is considered to be a vocally demanding task and thus places stress upon the vocal folds, the laryngeal muscles respond by increasing activity and increasing muscle tone. This increased muscle tone and activity reduces the degree to which cyclical variation would occur, thus resulting in decreased levels of jitter and shimmer [15].

Based on prior data, a few speculations may be offered concerning the current study. It is hypothesized that the majority of participants will exhibit an increase in $\mathrm{F}_{0}$ in conjunction with the vocal loading effect associated with

Table 1. Participant Information According To Age, Body Mass Index (BMI), Neck Fat (in mm), and the Initial and Final Level of Stimulation Intensity (in mA) Levels Used During TES

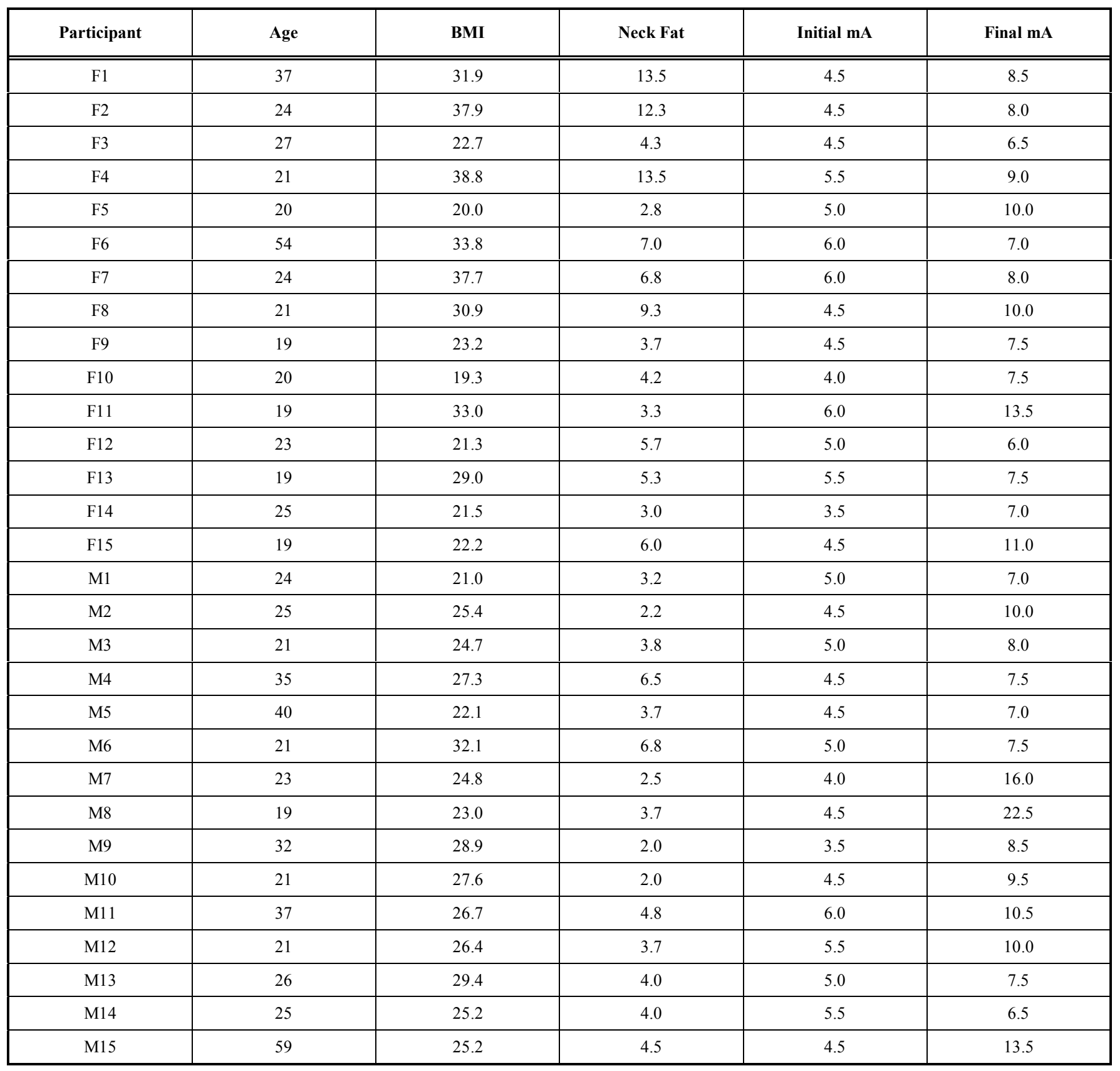


TES, while RLL may be vary in direction. It is also hypothesized the participants will exhibit a decrease in perturbation noise measures as an indicator of increased laryngeal muscle tone and activity due to the vocal loading effect. The decrease in cyclical variability, as represented by the perturbation measures, would likely contribute to an increase in SNR, as excessive variability in vocal fold vibration contributes to noise in the voice signal and is perceived as hoarseness or breathiness $[16,17]$.

\section{MATERIALS AND METHODOLOGY}

Participants. Fifteen women, ages 19 to 54 years, and 15 men, ages 19-59 years, were recruited as participants following approval of the study by the Valdosta State University Institutional Review Board. All participants signed an informed consent prior to enrollment in the study. None of the participants received monetary compensation for their participation in the study. Specific participant information is provided in Table 1. All participants were nonsmokers in good physical health, with no history of respiratory, cardiovascular, or neurological disorder. Participants also reported no history of speech, language, hearing, or voice difficulties. All participants demonstrated voice quality within normal limits, as judged by a certified speech-language pathologist, and were free from allergies or colds on the days of testing per self-report. Hearing acuity was informally judged to be within functional limits.

Electrode Placement. The placement of two pairs of bipolar surface electrodes (VitalStim ${ }^{\circledR}$, REF 59000, Chattanooga Group, Chattanooga, TN) was determined from pilot data which indicated that the greatest voice change occurred with electrodes placed in the anterior neck and submentally [18]. As previously reported by Fowler et al. [11], the first pair of electrodes was placed on either side of the thyroid lamina, just below the thyroid notch. The thyroid notch was palpated to determine the level of placement. The second pair of electrodes was placed in the submental region, lateral to midline. Both areas were wiped with an alcohol pad first, followed by a TENS Clean-Cote ${ }^{\circledR}$ Skin Wipe (Tyco Unit-Patch Model UP220, Uni-Patch, Wabasha, MN) to insure adherence of the electrodes to the skin. All male participants were clean shaven to allow for maximum contact between the electrode and the skin. For some participants, additional taping was required to maintain contact between the skin and the electrodes.

Transcutaneous electrical stimulation. All participants received one hour of TES using the VitalStim ${ }^{\circledR}$ unit, as described previously by Fowler et al. [11]. The VitalStim ${ }^{\circledR}$ device has a fixed $80 \mathrm{~Hz}$ pulse rate and produces fixed biphasic pulse durations of 700 microseconds. The intensity of stimulation was gradually increased in 0.5 milliamperes $(0.5$ $\mathrm{mA}$ ) until the participant reported a "grabbing" sensation. The "grabbing" sensation indicates perceptual evidence of muscle contraction and reflects the minimum "motor" level of stimulation (VitalStim ${ }^{\circledR}$ Training Manual). The initial level of stimulation was determined individually for each participant, based on the participant's report of a "grabbing" sensation. As an adaptation effect may occur over time, all participants were instructed to increase the level of stimulation as warranted to insure that they continued to feel the "grabbing" sensation. Intensity of stimulation was noted at both the onset and conclusion of TES (see Table 1). This procedure reflects typical recommendations for a dysphagia therapy session per VitalStim ${ }^{\circledR}$ guidelines. All participants engaged in conversation, eating, and drinking for the duration of the hour-long stimulation.

Voice recordings. Voice recordings were obtained within 5 minutes pre- and post-stimulation. All recordings were completed in a sound-treated booth using an AT 3032 omnidirectional condenser microphone at a distance of 30 $\mathrm{cm}$. Participants were asked to sustain the vowel /a/ for approximately 3-5 seconds and read the first two sentences of the Rainbow Passage [19] at a comfortable pitch and loudness level. Three trials of each task were completed. All samples were digitized directly into a desktop computer.

Subjective Report. All participants were asked to provide subjective comments regarding voice quality and laryngeal sensations. These comments were obtained 5 minutes postand again 24-48 hours post-TES to check for delayed onset muscle soreness (DOMS). The phenomenon of DOMS is defined as the sensation of pain and stiffness in the muscles that occurs from one to five days following unaccustomed or eccentric exercise $[20,21]$.

Physical Measurements. Body mass index (BMI) was calculated for each participant based on height and weight measurements. Neck fat measurements were also obtained for each participant using a Lange skinfold caliper. Measurements were taken just lateral to the thyroid notch. The female subjects exhibited higher $B M I$ values, ranging from 19.3 to 38.8 , with a mean of 27.3. For men, $B M I$ ranged from 21.0 to 32.1 , with a mean of 26.4. As would be expected, neck fat measurements were higher for women, ranging from 2.8 to $13.5 \mathrm{~mm}$ with an average of $6.7 \mathrm{~mm}$. Neck fat measurements for the male participants ranged from 2.0 to $3.8 \mathrm{~mm}$, with an average of $3.8 \mathrm{~mm}$ (see Table 1).

Analysis. For the sustained vowel task, the middle one second of phonation was hand-marked for analysis. Measures of Fundamental frequency $\left(F_{0}\right)$, Jitter, Shimmer, Signal-to-noise ratio (SNR), and Relative loudness level $(R L L)$ were obtained from each vowel segment. For the reading task, measures of Speaking fundamental frequency $(S F F$; the average fundamental frequency measured during connected speech) and Relative loudness level (RLL-pa) were obtained. All acoustic analyses were completed using TF32 [22]. Individual and group averages were calculated for all measures pre- and post-stimulation. Data for each measure were statistically analyzed separately using a Wilcoxon signed ranks test, with a Bonferroni corrected alpha level of $p \leq .007(.05 / 9=.005)$ [23].

Inter- and intra-rater reliability data were obtained on $20 \%(n=6)$ of the data for each parameter and analyzed using Spearman's rho correlation measure. Intra-rater reliability was high, ranging from $r_{s}=.924$ to $r_{s}=1.00$. Inter-rater reliability was also high, ranging from $r_{s}=.964$ to $r_{s}=1.00$.

\section{RESULTS}

Inspection of the data showed that the magnitude and direction of change for all parameters was variable. Specifically, a portion of the participants would exhibit an increase in a selected parameter following TES while others would exhibit a decrease in the same parameter. In addition, 
the direction of change for a particular participant varied among the various parameters. Since the purpose of the present study was to determine if the application of TES resulted in changes in acoustic parameters, all data were converted to absolute values and subsequently analyzed.

Acoustic Measurements. An initial correlation analysis demonstrated that sex was weakly correlated with the magnitude of change in fundamental frequency for both the sustained vowel task $\left(r_{s}=-.528, p=.003\right)$ and the Rainbow passage $\left(r_{s}=-.439, p=.015\right)$. These data were therefore analyzed separately for male $v s$ female participants.

As demonstrated in Figs. $(\mathbf{1}, \mathbf{2})$, measurable changes were observed in the acoustic parameters but were highly variable

a.

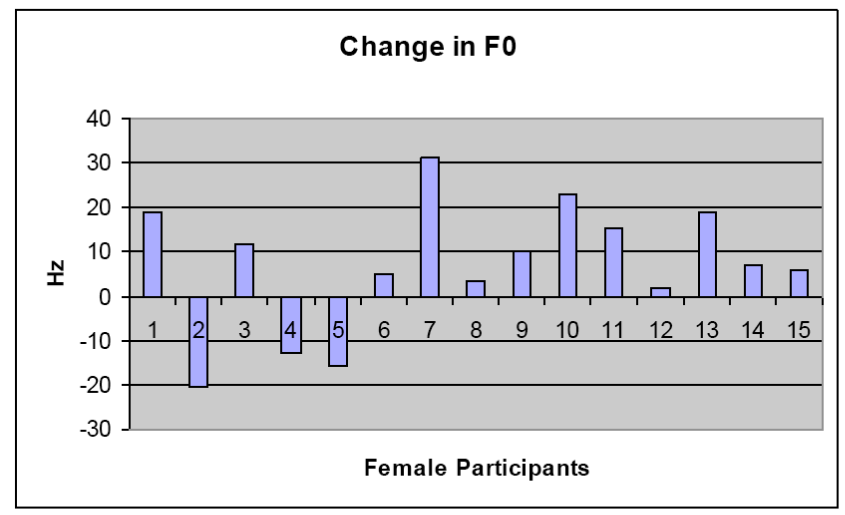

c.

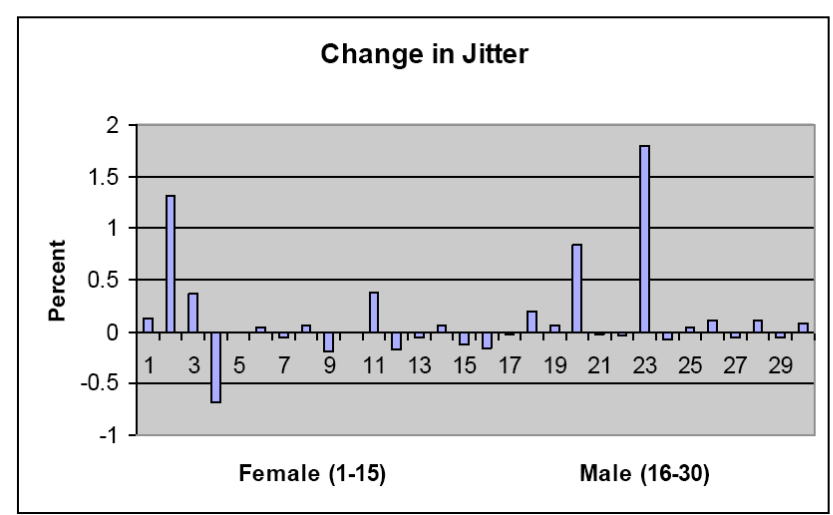

e.

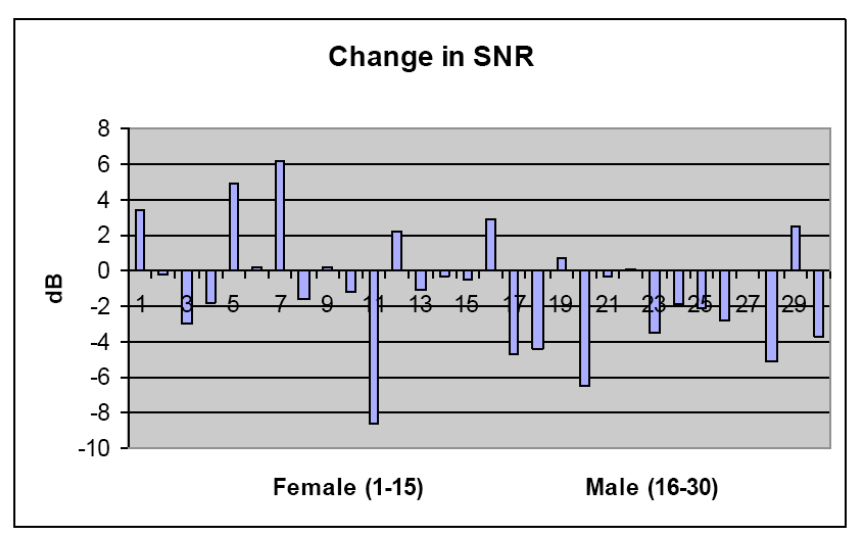

in magnitude and direction. The results of the Wilcoxon signed ranks test revealed that none of these changes were significant (see Table 2). However, there was a trend for increased shimmer and a slight increase in $R L L-p a$ following TES, as well as higher $S F F$ values for the women.

Subjective Comments. Examination of the remarks provided by the participants at the conclusion of TES revealed that a majority of the participants experienced a benign reaction to TES, either reporting no perceivable effect on voice or muscular activity ( $\mathrm{n}=6$, subsequently to be noted as group 1) or a vocal warm-up feeling $(\mathrm{n}=13$, group 2$)$. However, seven of the participants reported symptoms of vocal fatigue (group 3), while four others noted evidence of

b.

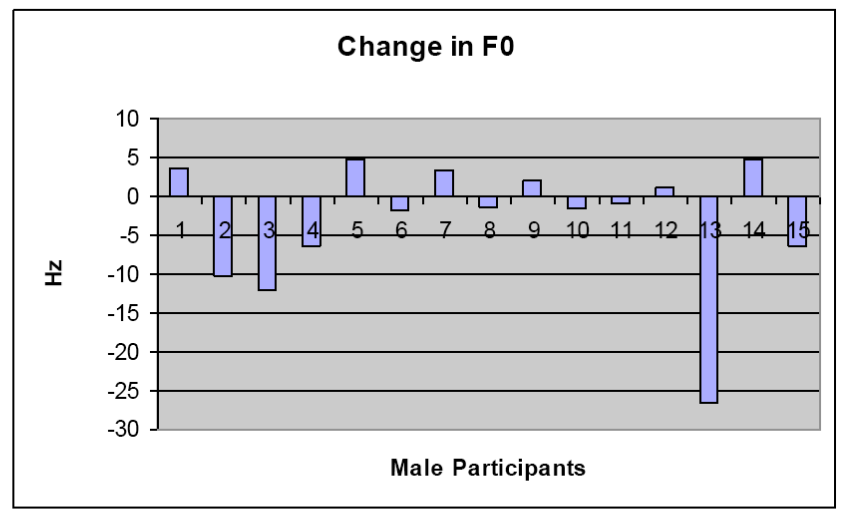

d.

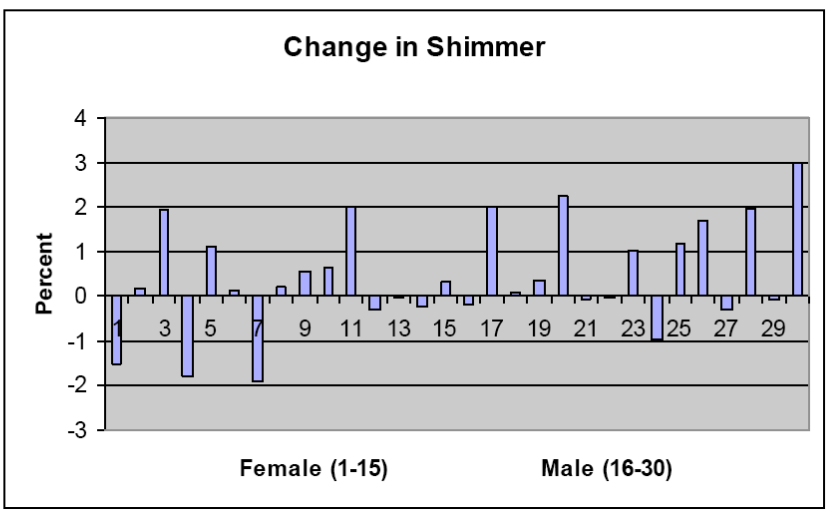

$\mathrm{f}$

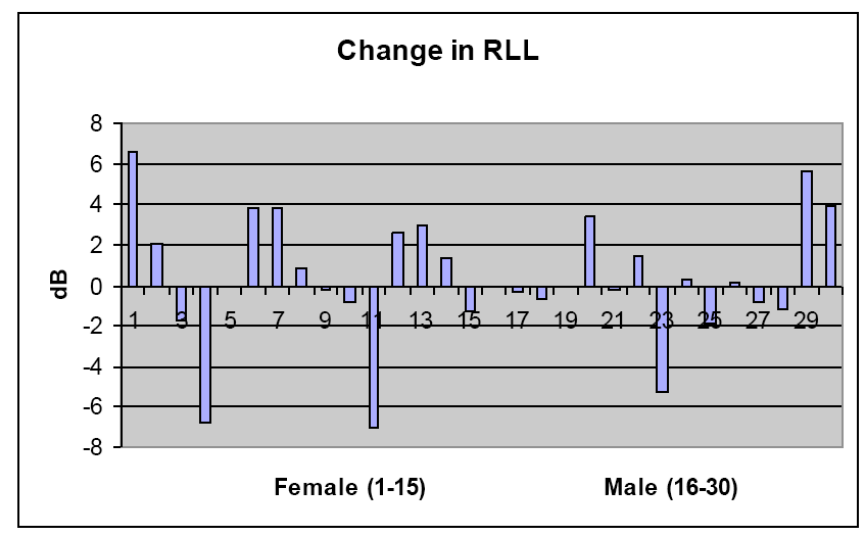

Fig. (1). (a-f) Direction and magnitude of change for the acoustic parameters measured during the sustained vowel production. Data are combined for all parameters for male and female participants except $\mathrm{F}_{0}$, as sex was correlated with $\mathrm{F}_{0}$. 
a.

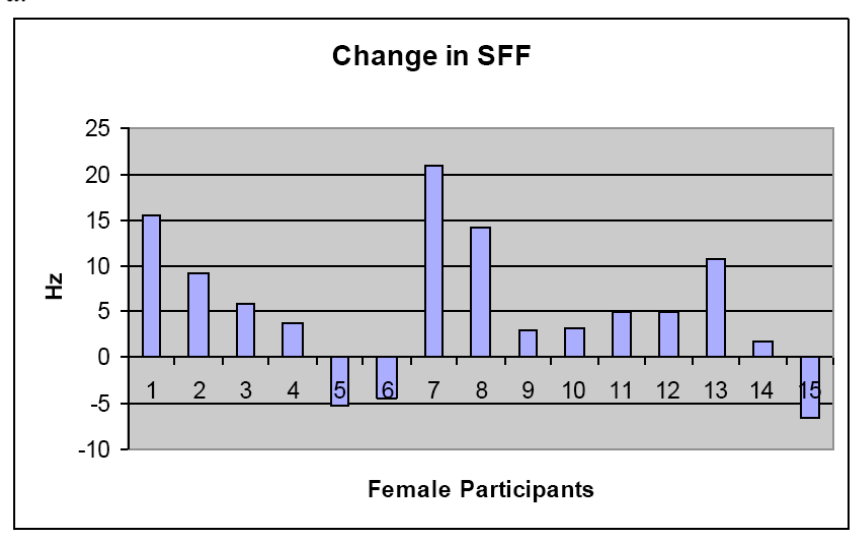

b.

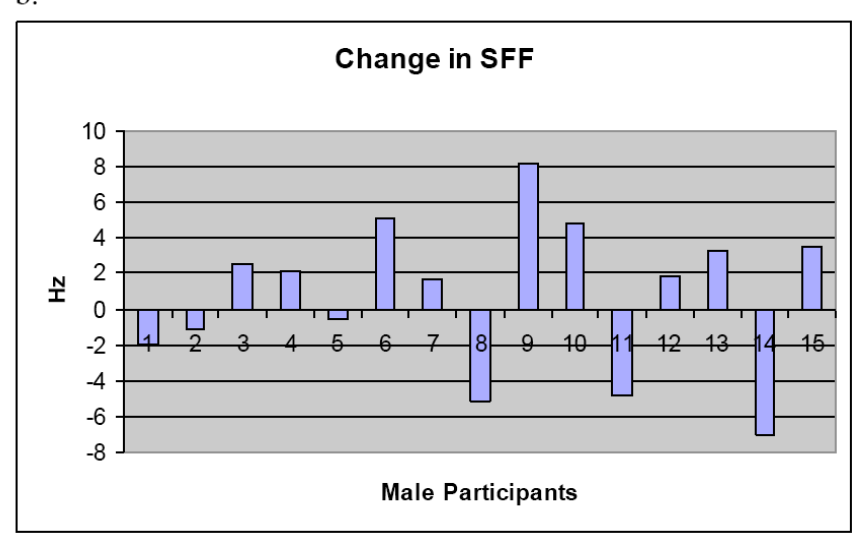

c.

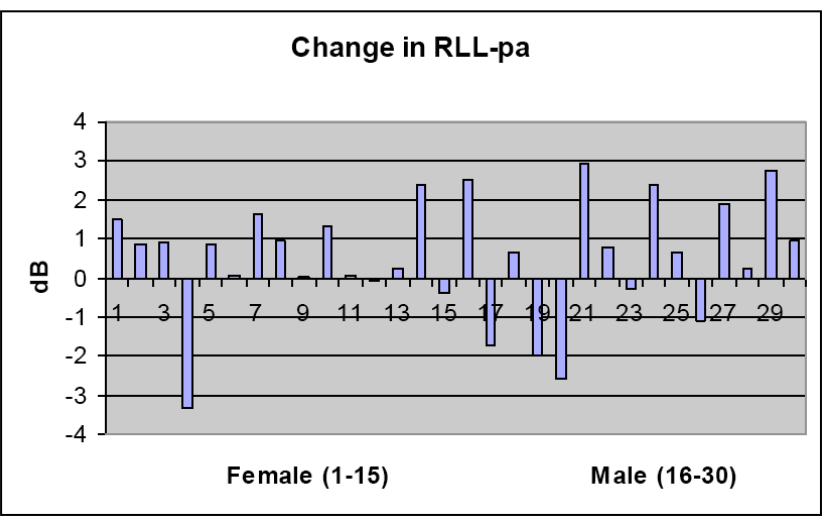

Fig. (2a-c). Direction and magnitude of change for the acoustic parameters measured during the Rainbow Passage. Data are displayed separately for female and male participants for SFF, as sex was correlated with SFF.

muscle injury e.g. soreness, tightness in the neck area (group 4). Examination of the data revealed that minimal difference between groups 1, 2, and 4 in the physical indices of age, $B M I$, and neck fat. There was also minimal difference in the magnitude of change in the acoustic parameters with the exception of SNR. Group 1 demonstrated a markedly greater decrease in $S N R$. In contrast, group 3 tended to be older and had higher neck fat measurements than the other groups. In addition, group 3 exhibited the smallest change in $F_{0}$ during the vowel production (see Table 3 ).

Table 2. Results of the Wilcoxon Signed-Ranks Test for the Dependent Measures

\begin{tabular}{|c|c|c|}
\hline Parameter & Wilcoxon & $p$-Value \\
\hline \multicolumn{3}{|l|}{ Sustained Vowel: } \\
\hline $\mathrm{F}_{0}$ change - women & -1.647 & 0.100 \\
\hline $\mathrm{F}_{0}$ change - men & -0.625 & 0.532 \\
\hline Jitter change & -0.746 & 0.455 \\
\hline Shimmer change & -1.985 & 0.047 \\
\hline SNR change & -1.806 & 0.071 \\
\hline RLL change & -0.876 & 0.381 \\
\hline \multicolumn{3}{|l|}{ Rainbow Passage: } \\
\hline SFF change & -2.211 & -0.027 \\
\hline RLL-pa change & -1.985 & 0.047 \\
\hline
\end{tabular}

A slightly different pattern emerged when examining comments provided by the participants 24 to 48 hours following TES. While many participants continued to report no effect $(\mathrm{n}=15$; group 1$)$ or feeling of being warmed up vocally $(\mathrm{n}=1$; group 2$)$, only two participants continued to report symptoms of vocal fatigue (group 3). However, a greater number of participants reported symptoms indicative of muscle injury at this time frame compared to 5 minutes post-TES ( $\mathrm{n}=10$; group 4). Participants in group 3 tended to be female, had the second highest neck fat measurements and exhibited the smallest change in RLL and RLL-pa (see Table 3).

\section{DISCUSSION}

The current study was completed in an attempt to extend initial findings concerning the effect of TES upon voice production in non-dysphonic speakers. The results of the study are in agreement with prior data demonstrating marked variability in the direction and magnitude of change in both $F_{0}$ and $R L L$, with the greatest magnitude of change occurring in $F_{0}$ [11]. These findings were present during both sustained vowel production and connected speech. As previously reported, the observed change is likely associated with recruitment of extrinsic laryngeal muscle, most probably that of the sternohyoid, sternothyroid, and/or thyrohyoid muscles due to the low current amplitude used by the participants and the superficial location of these muscles on the neck $[8,11]$.

This supposition of extrinsic muscle recruitment is strengthened by the finding that there were no significant 
Table 3. Physical Data and Magnitude of Change for the Acoustic Measures According to Groupings Based on Subjective Comments

\begin{tabular}{|c|c|c|c|c|c|c|c|c|c|c|}
\hline \multirow[b]{2}{*}{ Group } & \multirow[b]{2}{*}{ Age } & \multirow[b]{2}{*}{ BMI } & \multirow{2}{*}{$\begin{array}{l}\text { Neck } \\
(\mathbf{m m})\end{array}$} & \multirow{2}{*}{$\begin{array}{c}\mathrm{F}_{0} \\
(\mathbf{H z})\end{array}$} & \multicolumn{6}{|c|}{ Mean Magnitude of Change } \\
\hline & & & & & $\begin{array}{c}\text { Jitter } \\
(\%)\end{array}$ & $\begin{array}{c}\text { Shimmer } \\
(\%)\end{array}$ & $\begin{array}{l}\text { SNR } \\
\text { (dB) }\end{array}$ & $\begin{array}{l}\text { RLL } \\
\text { (dB) }\end{array}$ & $\begin{array}{l}\text { SFF } \\
\text { (Hz) }\end{array}$ & $\begin{array}{l}\text { RLL-pa } \\
\text { (dB) }\end{array}$ \\
\hline \multicolumn{11}{|l|}{5 Min. Post } \\
\hline 0 & 25.0 & 27.85 & 5.33 & -3.64 & 0.18 & 0.11 & -0.86 & 0.96 & 4.67 & 1.13 \\
\hline 1 & 25.9 & 26.48 & 4.56 & 3.70 & 0.29 & 0.95 & -2.61 & -0.66 & 4.11 & 0.57 \\
\hline 2 & 29.9 & 29.37 & 7.31 & 1.60 & -0.09 & -0.25 & 0.92 & 1.16 & 3.45 & -0.27 \\
\hline 3 & 26.3 & 24.03 & 3.92 & 4.51 & -0.08 & 0.51 & 0.69 & 1.35 & -2.72 & 1.06 \\
\hline \multicolumn{11}{|l|}{ 24-48 Hrs Post } \\
\hline 0 & 29.2 & 27.69 & 4.40 & 2.80 & 0.11 & 0.67 & -1.86 & 1.12 & 3.91 & 0.62 \\
\hline 1 & 20.3 & 25.67 & 3.11 & -0.64 & 0.60 & 0.64 & -1.85 & -2.63 & 0.54 & 0.75 \\
\hline 2 & 20.5 & 29.40 & 8.17 & -14.32 & -0.68 & -0.34 & 1.55 & -3.38 & -0.70 & -1.24 \\
\hline 3 & 26.1 & 26.19 & 6.65 & 4.41 & 0.13 & 0.20 & 0.05 & 0.86 & 3.57 & 0.61 \\
\hline
\end{tabular}

differences in the perturbation measures following TES. Since both jitter and shimmer reflect cycle-to-cycle variability in vocal fold vibration, such measures would more directly reflect intrinsic muscle activation. The lack of significant change in these measures suggests minimal effect of TES upon intrinsic laryngeal muscle control of vocal fold function when using low current amplitudes. Indeed, examination of the data reveals that nearly all participants maintained jitter and shimmer levels within normal limits following TES. Only one participant exhibited higher than normal jitter after TES (M5; jitter $=1.25 \%$ ), and only 5 of the participants demonstrated post-stimulation shimmer values that were considered above normal (F3, M5, M9, M10, M11, M15; shimmer $=5.26$ to $6.75 \%$ ). However, only one of these participants (M11) reported symptoms indicative of DOMS at 5 minutes and 24 hours poststimulation. The remaining participants reported a feeling of vocal warm-up or no sensations.

A similar pattern emerged in examining the data for $S N R$. Although over half of the participants exhibited a decrease in $S N R$ following TES, suggesting increased noise in the voice, only five participants (F8, F11, M9, M10, M13; $S N R=13.40$ to $24.17 \mathrm{~dB}$ ) reported symptoms associated with muscle injury. It is notable that $S N R$ values for most of the participants were within normal limits (19-20 dB or greater). Only M9 and M10 exhibited an SNR value below normal limits. Reasons for the negative symptoms reported by these two participants are unclear, as they did not differ greatly from many other participants in terms of age, BMI, neck fat, or initial level of stimulation intensity.

Examination of the subjective comments provided by the participants revealed a continuum of perceptual responses to TES, ranging from no sensation to symptoms coincident with DOMS. These findings are in agreement with previously reported data [11]. Reasons for the observed patterns are unclear, but a few speculations may be offered at this time. Just over half of the participants in the study reported no perceivable effect or a feeling of vocal warm-up immediately following and 24 to 48 hours post-TES. For these participants, it may be that as the stimulation continued, less current was directed towards the extrinsic muscles due to increased blood flow in the skin beneath the electrodes. As discussed by Fowler et al. [11], warm-up increases temperature and decreases muscle tissue viscosity [24]. Although not measured, it is possible that skin temperature also increased during the period of stimulation, which may require an increase in current intensity to provide the same stimulation [25]. While these participants did increase current intensity over the duration of the TES period, this increase was not remarkably different from the other groups. Additional factors may therefore have contributed to the perception of vocal warm-up or the lack of perceivable effects.

The reports of vocal fatigue by two women are supported by the observation that these participants exhibited the smallest change in both $\mathrm{F}_{0}$ and SFF. While an increase in fundamental frequency and/or loudness has been shown to occur in response to a vocal loading task, it is thought that minimal change or a decrease in either fundamental frequency and/or loudness is associated with vocal fatigue. The lack of change in these acoustic parameters reflects an inability of the laryngeal musculature, either intrinsic or extrinsic, to respond to a constant loading upon the vocal mechanism $[26,27]$. In the present study, 12 participants exhibited a decrease in $F_{0}$ during the sustained vowel task and nine exhibited a decrease in $S F F$ during the paragraph reading. However, these participants were more likely to report symptoms of vocal fatigue $(49 \%)$ compared to the participants who exhibited an increase in $F_{0} / S F F(31 \%)$.

The finding of vocal fatigue and/or DOMS may be related to the possibility that some of the women may have maintained higher levels of infrahyoid muscle activity following cessation of TES. Dietrich [28] reported higher levels of infrahyoid muscle activity following a simulated public speaking task in women identified as introverts compared to those classified as extroverts. All women in Dietrich's study exhibited a normal voice quality and had no history of voice difficulties. It is possible that individual 
differences in response to vocal stress may be a contributing factor to the development of voice difficulties. For example, Hočevar-Boltežar, Janko, \& Žargi [29] reported EMG evidence of increased muscle activity in the infrahyoids and the platysma in individuals with muscle tension dysphonia. Although personality traits were not examined in this study, it is possible that a portion of the women exhibited greater muscle tension in the infrahyoid musculature prior to TES, and this muscle activity increased following cessation of TES. Application of TES to the infrahyoid musculature may act as an external stressor, thus contributing to higher resting levels of muscle activity even when it is discontinued.

One must also consider the influence of physical factors such as BMI and neck fat upon muscular response to TES. Group 2 participants tended to have the highest $B M I$ and neck fat measurements of all groups, while those individuals included in group 3 at 24 to 48 hours post-stimulation demonstrated the second highest neck fat. Subcutaneous fat acts as a capacitor, thus storing the energy of the electrical current rather than transmitting it to deeper muscle layers [30]. Additionally, the type of wave used to transmit the current interacts with the amount of subcutaneous fat. Petrofsky [30] noted that an increase in subcutaneous fat creates impedance to the current flow when transmitted by a square waveform. The current flow is reflected back towards the skin rather than being transmitted towards the targeted muscles, thus causing greater discomfort. Given that the TES unit used in this study emits a biphasic square waveform pulse, it is conceivable that some of the participants with higher neck fat measurements actually experienced superficial skin discomfort rather than actual muscle fatigue or injury. Fifty percent of the participants in groups 2 and 3 had high amounts of neck fat. This scenario may partially explain why the acoustic measures failed to correlate with the subjective reports of the participants. Many of the participants who reported symptoms of DOMS did not show marked changes in the acoustic measures, while those individuals who reported no effect or a vocal warm-up feeling demonstrated greater instability and noise in the voice. The extent to which their symptoms reflect actual muscle fatigue or injury $v s$ superficial skin discomfort is not known.

\section{CONCLUSION}

The results of the present study demonstrate that the use of TES may result in changes in $F_{0}$ and loudness in individuals with normal voice quality. However, the response to TES is highly variable, both with regard to vocal changes as well as physical sensations. Some possible factors, such the amount of subcutaneous tissue in the anterior neck, individual response to hyolaryngeal lowering, and baseline infrahyoid activity, may account for this variability. Further research is warranted to determine other factors that may affect an individual's response to TES, including those individuals with voice difficulties as well as speakers with normal voice quality. Given the reports of vocal fatigue and DOMS, future studies should focus on the appropriate use of TES to minimize or eliminate these issues. At this time, current results indicate that the short-term effects (1 hour) of TES upon vocal fold vibratory behavior appear to be negligible. Findings regarding its application over time, or in conjunction with traditional therapeutic interventions, have not been published. As such, the effectiveness of the clinical application of TES to the neck, aside from addressing swallowing difficulties, remains to be proven.

\section{REFERENCES}

[1] Blumenfeld L, Hahn Y, LePage A, et al. Transcutaneous electrical stimulation $v s$ traditional dysphagia therapy: a noncurrent cohort study. Otolaryngol Head Neck Surg 2006; 135: 754-7.

[2] Ludlow CL, Humbert I, Saxon K, et al. Effects of surface electrical stimulation both at rest and during swallowing in chronic pharyngeal dysphagia. Dysphagia 2007; 22: 1-10.

[3] Kiger M, Brown CS, Watkins L. Dysphagia management: An analysis of patient outcomes using VitalStim ${ }^{\text {TM }}$ therapy compared to traditional swallow therapy. Dysphagia 2006; $21: 243-53$.

[4] LaGorio L, Carnaby-Mann G, Crary M. Cross-system effects of dysphagia treatment on dysphonia: a case report. Cases J 2008; 1: 67.

[5] Bülow M, Olsson R, Ekberg O. Videomanometric analysis of supraglottic swallow, effortful swallow, and chin tuck $\mathrm{n}$ healthy volunteers. Dysphagia 1999; 14: 67-72.

[6] Hind JA, Nicosia MA, Roecker EB, et al. Comparison of effortful and noneffortful swallows in healthy middle-aged and older adults. Arch Phys Med Rehab 2003; 82: 1661-5.

[7] Humbert IA, Poletto CJ, Saxon KG, et al. The effect of surface electrical stimulation on hyo-laryngeal movement in normal individuals at rest and during swallowing. J Appl Phys 2006; 101: 1657-63.

[8] Humbert IA, Poletto CJ, Saxon KG, et al. The effect of surface electrical stimulation on vocal fold position. Laryngoscope 2008; 118: 14-9.

[9] Erickson D, Baer T, Harris KS. The role of the strap muscles in pitch lowering. In: Bless DM, Abbs JH, Eds. Vocal Fold Physiology: Contemporary Research and Clinical Issues. San Diego (CA) USA: College-Hill 1983.

[10] Faaborg-Anderson K, Sonninen A. Function of the extrinsic laryngeal muscles at different pitches. Acta Otolarygol (Stockh) 1960; 51: 89-93.

[11] Fowler L, Gorham-Rowan M, Hapner E. An exploratory study of voice change associated with healthy speakers following transcutaneous electrical stimulation to laryngeal muscles. J Voice [in press].

[12] Hong KH, Ye M, Kim YM, et al. The role of strap muscles in phonation - in vivo canine model. J Voice 1997; 11: 23-32.

[13] Ferrand CT. Speech Science: An Integrated Approach to Theory and Clinical Practice. $2^{\text {nd }}$ ed. Boston (MA): Allyn \& Bacon 2007.

[14] Nittrouer S, McGowan RS, Milenkovic PH, et al. Acoustic measurement of men's and women's voices: A study of context effects and covariation. J Speech Hear Res 1990; 33: 761-75.

[15] Laukkanen A-M, Ilomaki I, Leppanen K, Vilkman E. Acoustic measures and self-reports of vocal fatigue by female teachers. J Voice 2008; 22: 283-9.

[16] Eskanazi L, Childers D, Hicks D Acoustic correlates of vocal quality. J Speech Hear Res 1990; 33: 298-306.

[17] Yumoto E, Gould WJ, Baer T. The harmonics-to-noise ratio as an index of degree of hoarseness. J Acous Soc Am 1982; 71: 1544-50.

[18] Gorham-Rowan M, Fowler L, Hapner E. A tutorial on muscle function and the effects of surface electrode stimulation on voice. Georgia Speech-Language-Hearing Association Convention. Atlanta (GA) 2006.

[19] Fairbanks G. Voice and Articulation Drillbook. $2^{\text {nd }}$ ed. New York (NY): Harper \& Row 1960.

[20] Armstrong, RB. (1984). Mechanisms of exercise-induced delayed onset muscle soreness: A brief review. Med Sci Sports Exerc 1984; 16: 529-38.

[21] Connolly DA, Sayers SP, McHugh MP. Treatment and prevention of delayed onset muscle soreness. J Strength Cond Res, 2003; 17 : 197-208.

[22] Milenkovic P. TF32. University of Wisconsin, Madison 2005

[23] Keppel G. Design and Analysis: A Researcher's Handbook. $2^{\text {nd }}$ ed. Englewood (NJ): Prentice-Hall 1982.

[24] Safran MR, Seaber AV, Garrett, WE. Warm-up and muscular injury prevention. An update. Sports Med 1989; 8: 239-49. 
[25] Petrofsky JS, Suh HJ, Gunda S, et al. Interrelationship between body fat and skin blood flow and the current required for electrical stimulation of human muscle. Med Eng Physics, 2008; 30: 931-6.

[26] Jónsdottir V, Laukkanen A-M, Siiki I. Changes in teachers' voice quality during a working day with and without electric sound amplification. Fol Phon Logop 2003; 55: 267-80.

[27] Rantala L, Vilkman E. Relationship between subjective voice complaints and acoustic parameters in female teachers' voices. J Voice 1996; 13: 484-95
[28] Dietrich M. The effects of stress reactivity on extralaryngeal muscle tension in vocally normal participants as a function of personality. PhD [dissertation]. Pittsburgh (PA): University of Pittsburgh 2008.

[29] Hočevar-Boltežar I, Janko M, Žargi M. Role of surface EMG in diagnostics and treatment of muscle tension dysphonia. Acta Otolaryngol 1998; 118: 739-43.

[30] Petrofsky J. The effect of subcutaneous fat on the transfer of current through skin and into muscle. Med Eng Phys 2008; 30: $1168-76$

(C) Gorham-Rowan et al.; Licensee Bentham Open

This is an open access article licensed under the terms of the Creative Commons Attribution Non-Commercial License (http: //creativecommons.org/licenses/by$\mathrm{nc} / 3.0 /$ ) which permits unrestricted, non-commercial use, distribution and reproduction in any medium, provided the work is properly cited. 